MATHEMATICAL ASSOCIATION

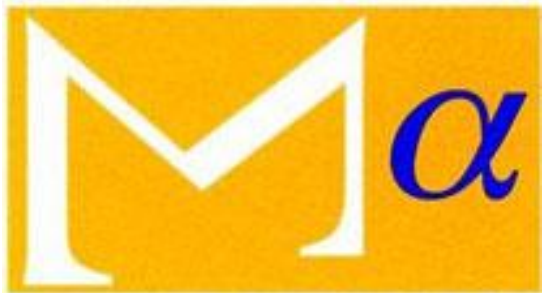

supporting mathematics in education

So-Called Cases of Failure in the Solution of Linear Differential Equations

Author(s): Eric H. Neville

Source: The Mathematical Gazette, Vol. 8, No. 123 (May, 1916), pp. 258-262

Published by: Mathematical Association

Stable URL: http://www.jstor.org/stable/3602942

Accessed: 03-01-2016 09:00 UTC

Your use of the JSTOR archive indicates your acceptance of the Terms \& Conditions of Use, available at http://www.jstor.org/page/ info/about/policies/terms.jsp

JSTOR is a not-for-profit service that helps scholars, researchers, and students discover, use, and build upon a wide range of content in a trusted digital archive. We use information technology and tools to increase productivity and facilitate new forms of scholarship. For more information about JSTOR, please contact support@jstor.org. 
For this part we have

$$
\int_{2000}^{3000}\left(-5+\frac{25 \cdot 1 \dot{6}}{10^{3}} x-\frac{3 \cdot 2}{10^{6}} x^{2}+\frac{0 \cdot 1 \dot{3}}{10^{9}} x^{3}\right) d x \text { pence, }
$$

which works out as $£ 165$. 18 s. 0 d.

Thus the amount of tax for $£ 3,000$ would be the sum obtained above for $£ 2,000$ plus $£ 165.18 s$. $0 d$.; in all, £323. 13s. $7 d$.

The other sums given in the Note to $(c)$ will be obtained in the same way.

We have dealt so far with the original Act. Two months after it became law, it was followed by an Amending Act, in which it was enacted that

"Where the income of a taxpayer consists of income from personal exertion and income from property the rates of the income tax shall be:

(a) In respect of the income from personal exertion-the rate that would have been applicable if the total taxable income of the taxpayer had been derived exclusively from personal exertion; and

(b) in respect of the income from property - the rate that would have been applicable if the total taxable income of the ratepayer had been derived from property."

I leave the interpretation of this to the industrious reader. His task would have been an easier one if what the Treasurer called "the beautifully simple terms of the mathematician" had received kinder treatment in the drafting of the original Act.

Sydney, N.S.W., Dec. 9, 1915.

\title{
SO-CALLED CASES OF FAILURE IN THE SOLUTION OF LINEAR DIFFERENTIAL EQUATIONS.
}

\author{
By Eric H. Neville.
}

(Reprinted from the Journal of the Indian Mathematical Society, vol.vii., 1915.)

There are two ways in which the solution of a particular linear differential equation may "fail" although the solution of a more general equation obtained by replacing certain constants by parameters is complete. The two ways are distinct, but they may both be illustrated by the equation

$$
(D-1)^{2} y=e^{x}
$$

where $D$ as usual stands for $d / d x$.

For the general equation

$$
(D-l)(D-m) y=e^{n x}
$$

the perfectly general solution is

$$
y=\frac{1}{(n-l)(n-m)} e^{n x}+A e^{l x}+B e^{m x},
$$

$A, B$ being independent arbitrary constants, but if we attempt to apply this solution to the particular equation (1), we find in the first place that the coincidence of $n$ with $l$ and $m$ renders the first term infinite, and in the second place that the coincidence of $m$ with $l$ leaves us with only one effective constant, $A+B$. The method by which in the commoner textbooks the passage from the general solution to that of a particular equation is made in such cases as this is unconvincing.

The fundamental problem is always the same: given a linear differential equation

$$
G(y)=g\left(x, a_{1}, a_{2}, \ldots a_{r}\right)
$$


of any order, $a_{1}, a_{2}, \ldots a_{r}$ being constants, $G(y)$ involving $x$ in any way and being homogeneous in $y$ and a finite number of its derivatives, and $g\left(x, a_{1}, a_{2}, \ldots a_{r}\right)$ being any function of $x, a_{1}, a_{2}, \ldots a_{r}$, and given a function $F\left(x, p_{1}, p_{2}, \ldots p_{r}\right)$ of $x$ and of $r$ parameters $p_{1}, p_{2}, \ldots p_{r}$ which is not infinite irrespectively of $x$ for the values $a_{1}, a_{2}, \ldots a_{r}$ of the paraneters and is such that

$$
G\left\{F\left(x, p_{1}, p_{2}, \ldots p_{r}\right)\right\}=h\left(p_{1}, p_{2}, \ldots p_{r}\right) f\left(x, p_{1}, p_{2}, \ldots p_{r}\right),
$$

where the right-hand side is not identically zero for all values of $p_{1}, p_{2}, \ldots p_{r}$ and for the values $a_{1}, a_{2}, \ldots a_{r}$ of $p_{1}, p_{2}, \ldots p_{r}$ the function $f\left(x, p_{1}, p_{2}, \ldots p_{r}\right)$ takes the form $g\left(x, a_{1}, a_{2}, \ldots a_{r}\right)$, to construct as general a solution as possible of the equation (2).

Thus in the original example we know that

$$
(D-1)^{2} e^{p x}=(p-1)^{2} e^{p x},
$$

and we wish to utilise this knowledge in the formation of integrals of the particular equation (1).

It will be noticed that here the function $f(x, p)$, which is $e^{p x}$, is actually the function $g(x, p)$; in practice this is usually the case, but it is quite irrelevant to the argument which follows, which enables us, for example, to deduce a solution of equation (1) from the more elaborate equation

$$
(D-1)^{2}\left(x e^{p x}\right)=2(p-1)\left\{e^{p x}+\frac{1}{2}(p-1) x e^{p x}\right\}
$$

taking for $f(x, p)$ the expression $\left\{e^{p x}+\frac{1}{2}(p-1) x e^{p x}\right\}$.

We consider first the construction of a particular integral of equation (2), equation (3) being assumed.

If $h\left(a_{1}, a_{2}, \ldots a_{r}\right) \neq 0$, we have from (3) and the known relation between the functions $f\left(x, p_{1}, p_{2}, \ldots p_{r}\right), g\left(x, a_{1}, a_{2}, \ldots a_{r}\right)$, and because the left-hand side is linear and homogeneous in $y$ and its derivatives,

$$
G\left\{F\left(x, a_{1}, a_{2}, \ldots a_{r}\right) / h\left(a_{1}, a_{2}, \ldots a_{r}\right)\right\}=g\left(x, a_{1}, a_{2}, \ldots a_{r}\right),
$$

and therefore

$$
y=F\left(x, a_{1}, a_{2}, \ldots a_{r}\right) / h\left(a_{1}, a_{2}, \ldots a_{r}\right)
$$

is a solution of (2). In any case, $p_{k}$ being any one of the parameters $p_{1}, p_{2}, \ldots p_{r}$, we have from (3) on account of the character of the operator $G$,

$$
G\left\{\partial F / \partial p_{k}\right\}=\left(\partial h / \partial p_{k}\right) f\left(x, p_{1}, p_{2}, \ldots p_{r}\right)+h\left(p_{1}, p_{2}, \ldots p_{r}\right)\left(\partial f / \partial p_{k}\right),
$$

and precisely when $h\left(a_{1}, a_{2}, \ldots a_{r}\right)$ is zero we can derive from (6) the $r$ equations

for $k=1,2, \ldots r$.

$$
G\left\{\partial F / \partial a_{k}\right\}=\left(\partial h / \partial a_{k}\right) g\left(x, \alpha_{1}, a_{2}, \ldots a_{r}\right),
$$

If then any of the functions $\partial h / \partial \alpha_{1}, \partial h / \partial a_{2}, \ldots \partial h / \partial a_{r}$ are not zero, solutions of the original equation (2) are given by

for the corresponding values of $k$.

$$
y=\frac{\partial F}{\partial a_{k}} / \frac{\partial h}{\partial a_{k}}
$$

Thus, from equation (5) we have

and from this it follows that

$$
\begin{aligned}
(D-1)^{2}\left\{\partial\left(x e^{p x}\right) / \partial p\right\} & =2\left\{e^{p x}+\frac{1}{2}(p-1) x e^{p x}\right\} \\
& +2(p-1)\left[\partial\left\{e^{p x}+\frac{1}{2}(p-1) x e^{p x}\right\} / \partial p\right],
\end{aligned}
$$

$$
y=\frac{1}{2}\left[\partial\left(x e^{p x}\right) / \partial p\right]_{p=1}=\frac{1}{2} x^{2} e^{x}
$$

is a solution of equation (1), the right-hand side of equation (7) reducing, for $p=1$, to a constant multiple of $e^{x}$ for the very reason that the factor $p-1$, which prevented our obtaining a solution of equation (1) by mere 
substitution in equation (5), remains a factor of that part of the expression which is not itself a constant multiple, for $p=1$, of $e^{x}$.

But it may happen that all the functions of the form $\partial h / \partial a_{k}$ are zero. For example, if we are using the simple equation (4) for the purpose of solving equation (1), the function $h(p)$ of the only parameter $p$ is $(p-1)^{2}$, and $d h / d p$ as well as $h(p)$ is zero when $p$ is equal to 1 . And in fact differentiation of the equation (4) with respect to $p$ gives us equation (5) itself, in the form

$$
(D-1)^{2}\left\{\partial e^{p x} / \partial p\right\}=2(p-1) e^{p x}+(p-1)^{2}\left(\partial e^{p x} / \partial p\right),
$$

and the right-hand side of this equation does not reduce to a proper multiple of $e^{x}$ for any value of $p$.

If, however, we differentiate the equation (6) with respect to any one of the parameters which it involves, we have for all values of the parameters and for all values of $i, k$ from 1 to $r$,

$$
G\left\{\frac{\partial^{2} F}{\partial p_{i} \partial p_{k}}\right\}=\frac{\partial^{2} h}{\partial p_{i} \partial p_{k}} f+\frac{\partial h}{\partial p_{i}} \partial p_{k}+\frac{\partial h}{\partial p_{k}} \frac{\partial f}{\partial p_{i}}+h \frac{\partial^{2} f}{\partial p_{i} \partial p_{k}}
$$

and when $h$ and its first derivatives with respect to the parameters $p_{i}, p_{h}$ (which may coincide) vanish for the values $a_{1} a_{2}, \ldots a_{r}$ of $p_{1}, p_{2}, \ldots p_{r}$, we have

$$
G\left\{\partial^{2} F / \partial a_{i} \partial a_{k}\right\}=\left(\partial^{2} h / \partial a_{i} \partial a_{k}\right) g\left(x, a_{1}, a_{2}, \ldots a_{r}\right)
$$

and if the second derivative $\partial^{2} h / \partial p_{i} \partial p_{k}$ of $h$ remains different from zero for the special values of the parameters, we can deduce a solution

$$
y=\frac{\partial^{2} F}{\partial a_{i} \partial a_{k}} / \frac{\partial^{2} h}{\partial \alpha_{i} \partial a_{k}}
$$

of our original equation. For example, from equation (8) we have

$$
(D-1)^{2}\left\{\partial^{2} e^{p x} / \partial p^{2}\right\}=2 e^{p x}+4(p-1)\left(\partial e^{p x} / \partial p\right)+(p-1)^{2}\left(\partial^{2} e^{p x} / \partial p^{2}\right)
$$

an equation which yields us a solution of the equation (1) in the form

$$
y=\frac{1}{2}\left(\partial^{2} e^{p x} / \partial p^{2}\right)_{p=1}=\frac{1}{2} x^{2} e^{x}
$$

simply because the earlier equations (4), (8) were ineffective for this purpose.

The reasoning can be continued from step to step, and we conclude that if $h\left(p_{1}, p_{2}, \ldots p_{r}\right)$ and all those of its partial derivatives of the first $m-1$ orders which are subsidiary to a particular derivative $\partial^{m} h / \partial p_{1}^{s_{1}} \partial p_{2}^{s_{2}} \ldots \partial p_{r}^{s_{r}}$ that is, which are of the form $\partial^{n} h / \partial p_{1}^{t_{1}} \partial p_{2}^{t_{2}} \ldots \partial p^{t_{r}}$, where $n<m, t_{k} ₹ s_{k}$, vanish when $p_{1}, p_{2}, \ldots p_{r}$ have the values $\alpha_{1}, a_{2}, \ldots a_{r}$, but this derivative itself does not then vanish, a particular solution of equation (2) is given by

$$
y=\frac{\partial^{m} F^{r}}{\partial a_{1}^{s_{1}} \partial \alpha_{2}^{s_{2}} \ldots \partial \alpha_{r}^{s_{r}}} / \frac{\partial^{m} h}{\partial a_{1}^{s_{1}} \partial \alpha_{2}^{s_{2}} \ldots \partial \alpha_{r}^{s_{r}}} .
$$

The common case is that in which there is only one parameter $p$, and only one constant $\alpha$ in the original equation, and the functions $f(x, p), g(x, p)$ are the same; $h$ is a function of $p$ alone, $F$ is a function of $x$ and $p$, and the result takes the form that if $h, d h / d p, d^{2} h / d p^{2}, \ldots d^{m-1} h / d p^{m-1}$ all vanish for $p=\alpha$, but $d^{m} h / d p^{m}$ does not then vanish, a solution of the equation

is given by

$$
G(y)=g(x, \alpha)
$$

$$
y=\frac{\partial^{m} F}{\partial a^{m}} / \frac{d^{m} h}{d a^{m}}
$$

$F(x, p)$ and $h(p)$ being such functions that

$$
G\{F(x, p)\}=h(p) g(x, p) .
$$


In this case we obtain only one solution of the equation, but in the general case we often obtain a number of solutions, which may or may not be independent.

To see how a multiplicity of parameters may be utilised, consider the equation

being given that

$$
(D-1)^{2}(D-2) y=e^{x}+e^{2 x},
$$

where

$$
(D-1)^{2}(D-2) F(x, p, q)=h(p, q)\left\{e^{p x}+e^{q x}\right\},
$$

$$
\begin{aligned}
F(x, p, q) & =(q-1)^{2}(q-2) e^{p x}+(p-1)^{2}(p-2) e^{q x}, \\
h(p, q) & =(p-1)^{2}(p-2)(q-1)^{2}(q-2) .
\end{aligned}
$$

If we make both $p=1$ and $q=2$ in $h(p, q)$ or in any of its derivatives of the first three orders except $\partial^{3} h / \partial^{2} \partial q$, the result is zero, but this one derivative has the value -2 . Hence one solution of equation (9) is given by

$$
y=-\frac{1}{2} \frac{\partial^{3} F(x, 1,2)}{\partial p^{2} \partial q}=-\frac{1}{2} x^{2} e^{x}+x e^{2 x} .
$$

It is easy to see that in this example no other integral is derivable from the functions $F(x, p, q), h(p, q)$.

For a second example, in which we cannot, without the use of complex numbers, resolve the problem into two simpler ones, as we should do in practice in the last example, we may solve the equation

$$
\left(D^{2}-2 D+2\right) y=e^{x} \sin x
$$

premising that

$$
\left(D^{2}-2 D+2\right) F(x, p, q)=h(p, q) e^{p x} \sin q x,
$$

where

$$
\begin{aligned}
F(x, p, q) & =e^{p x}\left[\left\{(p-1)^{2}+\left(1-q^{2}\right)\right\} \sin q x-2(p-1) q \cos q x\right], \\
h(p, q) & =\left\{(p-1)^{2}+\left(1-q^{2}\right)\right\}^{2}+4(p-1)^{2} q^{2} \ldots \ldots \ldots \ldots \ldots \ldots \ldots \ldots \ldots \ldots \ldots \ldots \ldots
\end{aligned}
$$

We see without difficulty that we may use either $\partial^{2} h / \partial p^{2}$ or $\partial^{2} h / \partial q^{2}$, and we find the two solutions

$$
\begin{aligned}
& y=\frac{1}{4} e^{x}(\sin x-2 x \cos x), \\
& y=-\frac{1}{4} e^{x}(\sin x+2 x \cos x),
\end{aligned}
$$

each of which as a matter of fact includes a part usually absorbed into the complementary function.

Two comments are suggested by this last example. In the first place, we do not pretend to have here an exceptionally simple way of solving the equation (10); we are concerned only to shew that if the equation itself is contained under a more general form of which we have the solution, a solution of the equation is obtainable by a direct and unimpeachable process. And in the second place, instead of starting from a known result involving two parameters, we might have assumed that

where

$$
\left(D^{2}-2 D+2\right) F(x, p)=h(p) e^{p x} \sin p x,
$$

$$
\begin{aligned}
F(x, p) & =e^{p x}(\sin p x+p \cos p x), \\
h(p) & =-2(p-1)\left(p^{2}+1\right), \ldots
\end{aligned}
$$

and obtained the slightly different solution

$$
y=-\frac{1}{4} e^{x}(\cos x+2 x \cos x) \text {. }
$$

A similar possibility is clearly open in general. All that is required of $p_{1}, p_{2}, \ldots p_{r}$ is that they should be capable of taking simultaneously the values $a_{1}, a_{2}, \ldots a_{r}$, and subject to this condition they may be made from the commencement any convenient functions of a single parameter $p$. Thus 
if $a_{1}, a_{2}, \ldots a_{r}$ are all different from zero we may put $p_{k}=a_{k} p$, and the particular equation to be studied will correspond to $p=1$, or in any case we may put $p_{k}=a_{k}+p$ in the general equation which is given to us and consider the effect of the vanishing of $p$. But, since different equations may most naturally be discussed in different ways, there is evident advantage in limiting ourselves as little as possible in the general treatment.

(To be continued.)

\section{MATHEMATICAL NOTES.}

467. [x. 7.] My son, Mr. E. Mann Langley, has pointed out to me a simple geometrical construction which yields a remarkable connexion between the centimetre and the inch.

Its simplest form of statement is as follows :

If the side of an equilateral triangle is 5 inches long, then (with a relative error less than 1 in 5000) its altitude is 11 centimetres long.

It may be utilised thus :

To obtain a scale of centimetres from a scale of $\frac{1}{2}$ inches.

Take two points $A$ and $B 2.5$ inches apart.

With centres $A$ and $B$ and radius 2.5 in. describe the semicircles $F B C D$, EFAC.

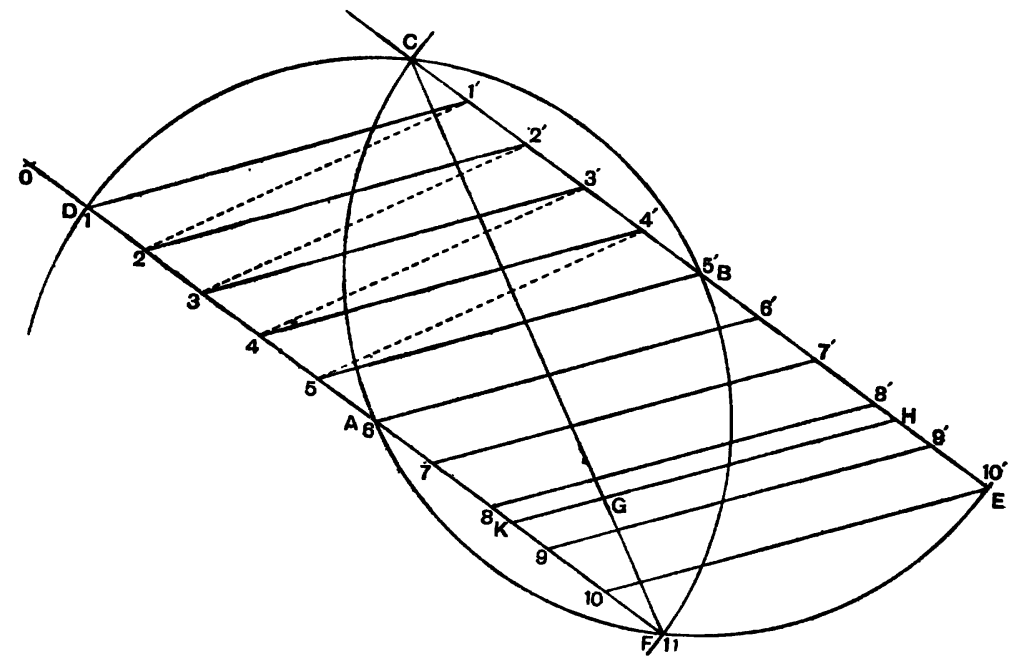

FIG. 1.

Graduate the diameters in $\frac{1}{2}$ inches as shown.

Then the lines $11^{\prime}, 22^{\prime}, 33^{\prime}$, etc. divide $C F^{\prime}$ into 11 equal parts indistinguishable from 11 centimetres on any ordinary scale.

$$
\begin{aligned}
& \text { For } \quad C F=2 \frac{1}{2} \times \sqrt{\overline{3}} \\
& =2 \frac{1}{2} \times 1 \cdot 7320508 \\
& =4 \cdot 330127 \\
& 10 \mathrm{~cm} .=3.937079 \\
& 1 \mathrm{~cm} .=\cdot 393708 \\
& 11 \mathrm{~cm} .=\overline{4 \cdot 330787} \\
& \frac{4 \cdot 330127}{.00066}
\end{aligned}
$$

Hence the relative error is less than $\cdot 00066 / 4 \cdot 33$, and equals 00015 approx. 